Revue européenne des sciences sociales

European Journal of Social Sciences

XL-124 | 2002

Histoire, philosophie et sociologie des sciences

\title{
Normes, communauté et intentionnalité
}

\section{Pierre Jacob}

\section{OpenEdition \\ Journals}

Édition électronique

URL : http://journals.openedition.org/ress/572

DOI : $10.4000 /$ ress.572

ISSN : 1663-4446

\section{Éditeur}

Librairie Droz

\section{Édition imprimée}

Date de publication : 1 août 2002

Pagination : 27-38

ISBN : 2-600-00806-3

ISSN : 0048-8046

Référence électronique

Pierre Jacob, « Normes, communauté et intentionnalité », Revue européenne des sciences sociales [En ligne], XL-124 | 2002, mis en ligne le 01 décembre 2009, consulté le 04 mai 2019. URL : http:// journals.openedition.org/ress/572; DOI : 10.4000/ress.572 
Pierre JACOB

\section{NORMES,
COMMUNAUTÉ ET INTENTIONNALITÉ}

Dans les pages suivantes, les mots «pensée » et «intentionnalité » seront interchangeables. Par «pensée» ou «intentionnalité», j'entends une représentation mentale d'un état de choses ayant conjointement un contenu conceptuel et une condition de vérité. De même que la signification complexe d'une phrase d'une langue naturelle dépend de la signification des expressions dont elle est composée et des règles grammaticales de la langue en question, de même une pensée possède un contenu conceptuel complexe qui dépend du contenu des concepts dont elle est formée et de sa structure logique. Je suppose qu'une représentation peut être mentale ou non mentale. L'évolution de la cognition humaine a donné naissance à une prolifération d'artefacts parmi lesquels certains sont des représentations non mentales. Les énoncés linguistiques, les symboles logiques et mathématiques, les diagrammes, les plans de construction, les panneaux routiers, les cartes géographiques, les tableaux, les dessins, les photographies, les films et les états des appareils de mesure (les jauges, les thermomètres, les échelles, les galvanomètres, etc.), les pensées, les jugements, les croyances, les désirs, les intentions, les expériences perceptives, les souvenirs, les images mentales sont des représentations mentales. Toute représentation mentale ou non possède un contenu ou l'intentionnalité. Comme l'a souligné Brentano, avoir l'intentionnalité ou posséder des concepts, c'est pouvoir représenter non seulement des choses qui existent dans l'espace et dans le temps, mais aussi des entités abstraites (qui, comme les nombres, ne sont ni dans l'espace ni dans le temps) et des entités fictives ${ }^{1}$.

Je souscris de surcroît à la distinction entre l'intentionnalité dite «primitive» des représentations mentales et l'intentionnalité dite «dérivée» des artefacts. Je suppose donc qu'un artefact tire son contenu du contenu des représentations mentales des agents humains qui s'en servent pour ébruiter et communiquer leurs propres pensées, croyances, intentions, et ainsi de suite ${ }^{2}$.

Enfin, je suppose que les représentations mentales et linguistiques peuvent avoir ce que Searle $(1983,2001)$ nomme deux directions d'ajustement (direction of fit): une direction d'ajustement esprit-monde et une direction d'ajustement monde-esprit. A la différence de l'énoncé d'une phrase impérative, l'énoncé

Dans ce qui suit, j’assimilerai intentionnalité et contenu conceptuel.

2 Cette distinction, qui est admise par des auteurs aussi différents que J. Haugeland, J.A. Fodor, F. Dretske et J. Searle, est contestée vigoureusement par D. Dennett (1987) dans The Intentional Stance. Je la défends dans les livres parus en 1997 What Minds Can Do et Pourquoi les choses ontelles un sens? 
d'une assertion possède une condition de vérité. A la différence d'une intention ou d'un désir, les croyances et les jugements possèdent une condition de vérité. Lorsqu'un individu énonce une phrase impérative, forme un désir ou une intention, son esprit ne s'ajuste pas au monde: il représente non pas un fait mais un but, c'est-àdire un état de choses possible ou impossible mais non réalisé. Mais lorsqu'un individu fait une assertion, forme une croyance ou porte un jugement, son esprit s'ajuste au monde: il cherche à représenter des états de choses réalisés ou des faits, c'est-à-dire le monde tel qu'il est. Dans ce qui suit, par les mots «pensée» ou «intentionnalité », je désignerai les croyances et les jugements, c'est-à-dire les représentations mentales ayant à la fois un contenu conceptuel et une condition de vérité - celles qui manifestent la direction d'ajustement de l'esprit au monde.

\section{LE PROBLÈME: L'INTENTIONNALITÉ EST-ELLE INTRINSÈQUEMENT SOCIALE?}

Parmi les propriétés et les activités que peut exemplifier un être humain, certaines sont intrinsèquement sociales en ce sens que l'appartenance à une communauté d'agents humains est une condition nécessaire à l'exemplification de ces propriétés ou au déroulement de ces activités. Une personne ne peut pas être mariée, divorcée ou veuve si sa relation à un partenaire n'a pas été sanctionnée selon des règles en vigueur dans une communauté. Faute d'appartenir à une communauté, une personne ne peut ni devoir, ni payer des impôts. Faute d'appartenir à une communauté de partenaires conscients, une personne ne peut ni danser la valse, ni assassiner, ni cambrioler, ni voter, ni recevoir un salaire, ni acheter une baguette, ni communiquer, ni a fortiori participer à une conférence scientifique. Le mariage, le divorce, le veuvage, les relations fiscales, la valse, les assassinats, les cambriolages, les processus électoraux, le salariat, les relations monétaires et la communication sont des propriétés, des relations et des activités intrinsèquement sociales.

Selon la conception externaliste du contenu des pensées d'un individu - de ses croyances et de ses jugements - à laquelle je souscris, nombreuses sont les pensées d'un individu dont le contenu dépend des relations entre, d'une part, le cerveau de cet individu et, d'autre part, des objets, des espèces naturelles, des propriétés et des relations exemplifiés dans l'environnement de cet individu ${ }^{3}$. Par exemple, faute d'être en relation avec des chevaux, un individu ne pourrait pas posséder le concept de cheval. Si les ancêtres de mes ancêtres n'avaient pas interagi avec des chevaux et si mon développement ontogénétique n'avait pas pris place dans un environnement contenant des chevaux, je serais privé du concept de cheval. La conception externaliste du contenu des pensées d'un individu s'oppose à une conception internaliste selon laquelle le contenu des pensées d'un individu dépend des seules ressources cognitives intrinsèques d'un individu, indépendamment de la nature de son environnement physique, chimique et biologique.

\footnotetext{
L'externalisme est défendu, par exemple, par H. Putnam (1975) dans The Meaning of 'Meaning' et par T. Burge (1979) dans Individualism and the Mental.
} 
J'admets aussi que nombre des concepts et des pensées d'un individu dépendent de facto de ce que pensent d'autres membres de sa communauté ${ }^{4}$. Je nommerai «déférentiels » les concepts qu'un individu forme grâce à la communication verbale et en vertu de son appartenance à une communauté. J'admets que la plupart de mes concepts scientifiques sont des concepts «déférentiels» en ce sens que je ne les possède que grâce à la communication verbale et par «déférence » à l'égard d'autres membres de ma communauté qui ont des compétences théoriques, techniques et expérimentales qui me font défaut. Je suppose par conséquent que la communication verbale et l'existence d'une communauté de personnes sont des conditions indispensables à la culture en général et à cette activité culturelle humaine particulière qu'est la démarche scientifique.

Mais la question que je veux soulever dans les pages qui suivent est la question de savoir si l'appartenance à une communauté est une condition nécessaire de jure de l'intentionnalité, de la pensée conceptuelle ou de la possession de concepts. La question que je vais examiner est la question de savoir si penser est une activité ou un processus intrinsèquement social. Selon une doctrine que je nommerai, en détournant le mot du sens que lui confère la philosophie politique contemporaine, «le communautarisme», faute d'appartenir à une communauté d'agents humains, un individu ne pourrait tout simplement pas penser, avoir des concepts ou avoir l'intentionnalité. Faute d'appartenir à une communauté, un individu ne pourrait former aucune représentation mentale conceptuelle digne d'être qualifiée de «pensée». Un partisan du communautarisme soutient, par exemple, qu'avant sa rencontre avec Vendredi, Robinson Crusoé ne pouvait pas penser, avoir des concepts ou qu'il était dépourvu de l'intentionnalité.

Une chose est de reconnaître que les concepts ésotériques exprimés par les mots français «lymphocyte», «lepton» ou «quark» sont des concepts déférentiels ou qu'une personne naïve qui n'est ni immunologiste ni physicien n'a acquis ces concepts et ne peut utiliser ces mots qu'en vertu de son appartenance à une communauté qui inclut des immunologistes et des physiciens qui connaissent les conditions d'emploi de ces mots. Autre chose est de proclamer avec les partisans du communautarisme que la pensée ou la possession de tout concept est une activité ou une propriété intrinsèquement sociale.

Quoique je souscrive à la conception externaliste du contenu des pensées, je ne souscris pas au communautarisme. En quelques mots, je ne souscris pas au communautarisme parce que je ne crois pas que la perception visuelle, auditive, olfactive ou tactile, la motricité et la planification de l'action sont des processus et des activités intrinsèquement sociaux. Si le communautarisme était vrai de la pensée ou de l'intentionnalité et s'il ne s'appliquait pas à la perception, à la motricité et à la planification de l'action, alors les processus conceptuels seraient séparés par un fossé infranchissable des processus perceptifs et moteurs. Dans les pages qui suivent, je veux examiner un argument fascinant présenté par le grand logicien et philosophe Saul Kripke en faveur d'une version du communautarisme dans son petit livre de 1982 intitulé Wittgenstein on Rules and Private Language.

4 Ce qu'on peut nommer «l'externalisme social» est défendu vigoureusement par T. Burge (1979) dans Individualism and the Mental. 
Cet argument procède en deux étapes. La première étape culmine dans ce que Kripke (1982) nomme le «paradoxe sceptique» dont il attribue la paternité à Wittgenstein (1953). Comme je le ferai valoir, le paradoxe sceptique est une version de la thèse selon laquelle la propriété sémantique d'une représentation, la signification d'un mot, le contenu d'un concept, la vérité d'une croyance sont des propriétés intrinsèquement normatives. En un mot, l'intentionnalité serait intrinsèquement normative. Ce qui serait constitutif de l'intentionnalité, c'est le pouvoir d'envisager ce qui doit être - le devoir être - et non ce qui est. Selon le paradoxe sceptique, en énonçant la signification d'un symbole (ou en attribuant une signification à un symbole), on ne décrit aucun fait sémantique, car les faits sémantiques n'existent pas. On exprime une norme. Autrement dit, on énonce ce qui doit être et non pas ce qui est ${ }^{5}$. La seconde étape consiste à offrir au «paradoxe sceptique» une solution que Kripke (1982) qualifie aussi de «sceptique» et dont il attribue également la paternité à Wittgenstein. Comme je le ferai valoir, la « solution sceptique » est une version du communautarisme. Elle affirme en effet que l'intentionnalité est intrinsèquement sociale.

On attribue à Hume le mérite d'avoir démontré que c'est un sophisme de chercher à dériver une obligation morale à partir de propositions de fait. Au début du siècle dernier, Moore a critiqué ce qu'il appelait «le sophisme naturaliste» qui consiste à essayer de définir le sens du mot évaluatif «bon» ou «bien» en termes purement descriptifs et non normatifs. $\mathrm{Si}$, comme l'affirme le paradoxe sceptique, les propriétés sémantiques d'une représentation sont intrinsèquement normatives, alors quiconque - comme moi - est engagé dans le projet d'une réduction naturaliste des faits sémantiques à des faits non sémantiques s'expose fatalement à l'accusation de commettre une nouvelle version du sophisme naturaliste ${ }^{6}$.

\section{LE PARADOXE SCEPTIQUE (OU L'INTENTIONNALITÉ EST INTRINSÈQUEMENT NORMATIVE)}

Qu'est-ce qu'un paradoxe? Un paradoxe est une proposition inacceptable ou incompatible avec des croyances bien établies (ou tenues pour telles). Quiconque envisage de rejeter une proposition paradoxale doit rejeter l'une des prémisses qui ont conduit à la conclusion paradoxale. J'ai expliqué ailleurs comment je propose

5 Nombre de philosophes contemporains éminents - dont D. Davidson et J. McDowell - souscriraient sans doute à une version de la thèse selon laquelle l'intentionnalité est intrinsèquement normative. Mais d'une part, dans Essays on Actions and Events, D. Davidson (1980), qui souscrit à une ontologie nominaliste, préfère s'abstenir de faire référence à des propriétés sémantiques. Il préfère dire que les objets et les événements physiques tombent sous des prédicats mentaux ou sémantiques. D'autre part, il invoque le rôle constitutif de la rationalité dans l'attribution d'attitudes propositionnelles à un agent humain. Dans Mind and World, J. McDowell (1994) oppose «le royaume des lois naturelles» et le «royaume des raisons» comme deux domaines irréductibles l'un à l'autre. Dans son célèbre Truth, M. Dummett (1978) a pour sa part soutenu que la vérité est une propriété normative d'une pensée ou d'un énoncé en faisant valoir que c'est une valeur à laquelle nous aspirons.

6 Pour une justification et une ébauche de ce projet, cf. mes livres What Minds Can Do et Pourquoi les choses ont-elles un sens? 
de rejeter le paradoxe sceptique, c'est-à-dire la thèse de la normativité intrinsèque de la signification ${ }^{7}$. La «solution sceptique» préconisée par Kripke (1982) - au nom de Wittgenstein (1953) - ne consiste pas à localiser une prémisse fautive dans le cheminement qui conduit au paradoxe. Dans un esprit wittgensteinien, elle consiste à apprendre à vivre avec la conclusion paradoxale. Ici, après avoir brièvement décrit les étapes qui conduisent à la formulation du paradoxe sceptique, je montrerai pourquoi la solution sceptique ne peut pas, selon moi, être une solution au paradoxe sceptique.

A la question arithmétique de savoir «Combien font $68+57$ ?», quiconque a comme moi - une maîtrise explicite ou implicite des axiomes de l'arithmétique peut fournir la réponse arithmétique qui, pour être naïve, n'en est pas moins correcte: « $125 »$. Non que la maîtrise des règles de l'addition garantisse la correction du résultat du calcul. La connaissance des règles de l'addition ne protège pas contre les erreurs de calcul: elle ne confère pas l'omniscience arithmétique. Aussi correcte soit-elle, ma réponse arithmétique naïve se prête à la question méta-arithmétique soulevée par l'interlocuteur sceptique imaginé par Kripke (1982): «Qu'est-ce qui justifie ma réponse arithmétique naïve?». A cette question, je serai enclin à fournir la réponse méta-arithmétique suivante: «Je connais la règle de l'addition arithmétique (ou la signification du signe arithmétique '+') que j'ai apprise à l'école et pour fournir la réponse à la question arithmétique posée, j'ai suivi cette règle». Ma réponse méta-arithmétique s'expose alors à l'objection sceptique de la finitude: dans le passé, je n'ai appliqué qu'un nombre fini d'exemples de la fonction d'addition. Supposons qu'avant de répondre à la question arithmétique posée, je n'avais jamais explicitement effectué l'addition $68+$ 57. Quoique par hypothèse je n'ai effectué qu'un ensemble fini d'additions, quiconque - comme moi - déclare connaître une règle prétend pouvoir l'appliquer à un ensemble potentiellement infini de cas. Qu'est-ce qui prouve, demande le sceptique, qu'en répondant « $125 »$, j'ai suivi les règles de l'addition et non pas les règles de calcul d'une fonction différente de l'addition ou d'une fonction non standard - que Kripke (1982) nomme la «quaddition »- telle que $\mathrm{x} \oplus \mathrm{y}=\mathrm{x}+\mathrm{y}$ ssi $\mathrm{x} \& \mathrm{y}<68$ et $\mathrm{x} \oplus \mathrm{y}=5$ autrement?

Croyant connaître la règle d'addition, je crois naïvement qu'en réponse à toute question arithmétique, je suis la règle de l'addition et me conforme à la signification du signe «+». Mais je n'ai aucun moyen d'exclure l'hypothèse qu'au lieu de suivre cette règle, $\mathrm{j}$ 'applique en réalité la règle de la fonction de quaddition et me conforme à la signification du signe «quus». Lorsque je crois suivre une règle pour répondre à une question arithmétique, en réalité, comme le dit Kripke (1982), «j'accomplis un acte de foi injustifié (...) j'énonce '125' sans hésiter, comme un automate. Mais si je n'ai jamais effectué explicitement ce calcul auparavant, j'aurais aussi bien pu répondre ' 5 '. Rien ne justifie une simple inclination brute à donner une réponse plutôt qu'une autre».

A la question de savoir en vertu de quels faits mentaux tirés de mon histoire psychologique il est vrai que lorsque j'utilise le signe «+», j'ai l'intention de faire référence à la fonction d'addition plutôt qu'à la fonction de quaddition, il est ten-

Dans mes articles Les propriétés sémantiques sont-elles intrinsèquement normatives? et dans Is Meaning Intrinsically Normative? 
tant d'offrir ce que Kripke nomme «la solution dispositionnelle». Selon la solution dispositionnelle, les conditions de vérité de l'affirmation selon laquelle par «+», je veux dire «plus» et non pas «quus», sont constituées par mes dispositions à répondre à n'importe quelle question arithmétique du type «Combien font $\mathrm{x}+\mathrm{y}$ ?». Mais à la solution dispositionnelle Kripke (1982) oppose l'objection sceptique normativiste selon laquelle le problème n'est pas de savoir ce que je répondrais mais ce que je dois répondre. Comme le dit Kripke (1982), «le dispositionnaliste offre une solution descriptive (...) Mais la solution recherchée est normative et non pas descriptive».

L'exemple arithmétique choisi par Kripke (1982) soulève peut-être certains problèmes particuliers. Mais pour mesurer la généralité du problème soulevé par le paradoxe sceptique, il suffit de prendre n'importe quel mot d'une langue naturelle. En vertu de sa signification, le mot anglais «goose» désigne des oies. En vertu de sa signification, le mot anglais «goose» doit donc être appliqué à des oies, et rien d'autre. L'appliquer à autre chose qu'à une oie, c'est commettre une erreur.

Le paradoxe sceptique consiste à affirmer qu'en attribuant une signification à un symbole (le signe «+»), on ne décrit aucun fait sémantique: on exprime une norme. Les attributions de signification n'ont pas de conditions de vérité: aucun fait ou état de choses de la réalité objective ne leur correspond. Elles n'ont que des conditions d'assertion ou d'assertibilité. Le paradoxe sceptique est aux propriétés sémantiques ce que la théorie émotiviste est aux propriétés éthiques. Selon l'émotivisme, en énonçant une phrase contenant une expression normative douée d'une signification évaluative éthique, on ne décrit aucun état de choses, on n'exprime aucune proposition vériconditionnelle. On se contente d'ébruiter une préférence ou une aversion subjective douée d'une signification «émotive » et dépourvue de signification « cognitive».

Le paradoxe sceptique n'est donc pas simplement une thèse épistémologique: c'est une thèse ontologique. Comme le dit Kripke (1982), «Dieu lui-même qui connaît tous les faits ne sait pas si je veux dire plus ou quus». Dans le panthéon des faits connus de Dieu - c'est-à-dire de la totalité métaphysique des faits - il n'y a aucun fait sémantique. En bref, le paradoxe sceptique nie l'existence des faits sémantiques et il découle de deux prémisses fondamentales: la prémisse de la finitude et la prémisse de la normativité. La prémisse de la finitude affirme qu'il existe un fossé infranchissable entre les usages finis d'un symbole et le fait qu'une règle sémantique s'applique à un ensemble infini de cas. Selon la prémisse de la normativité, si le symbole «+» signifie la fonction d'addition (ou fait référence à l'ensemble de tous les triplets de nombres entiers qui sont dans la relation $<\mathrm{x}+\mathrm{y}=\mathrm{z}>$ ), alors ce symbole doit être utilisé pour désigner un triplet de nombres entiers bien déterminé. Le conséquent de la prémisse conditionnelle normative ne décrit aucun état de choses réalisé; il énonce ce qui doit être. L'interlocuteur sceptique imaginé par Kripke (1982) se sert de la prémisse de la normativité pour exiger une réponse normative à la question méta-arithmétique: «Que dois-je répondre à une question arithmétique du type ' $\mathrm{x}+\mathrm{y}$ ?'», par opposition à la question méta-arithmétique dispositionnelle "Que suis-je disposé (ou enclin) à répondre à une question arithmétique du type ' $\mathrm{x}+\mathrm{y}$ '?».

8 Théorie notamment défendue par A.J. Ayer (1936) dans Language, Truth and Logic. 
La prémisse de la finitude me paraît incontestable. Mais de ce que la signification d'un symbole a des conséquences normatives, il ne s'ensuit pas ipso facto qu'il n'existe pas de faits sémantiques ${ }^{9}$. C'est un fait que le mot anglais «goose» signifie la même chose que le mot français «oie». C'est un fait qu'il s'applique à des oies ou exprime le concept d'oie. Il suit de ce fait qu'il doit être appliqué à des oies et non à autre chose ${ }^{10}$. En général, du fait que l'exemplification d'une propriété ou d'une relation a des conséquences normatives, il ne s'ensuit pas que cette propriété ou cette relation est elle-même intrinsèquement normative. La relation exprimée par le verbe français «tuer» est sans doute une relation naturelle non normative: si $x$ tue $y$, alors $y$ cesse de vivre, c'est-à-dire de fonctionner comme un être vivant. Mais si cette relation est exemplifiée et si ses relata, $x$ et $y$, sont un agent et un patient humains, alors cette exemplification se prête à une évaluation normative et l'agent doit être sanctionné. Autrement dit, «du fait» que l'exemplification d'une propriété sémantique a des conséquences normatives, il ne s'ensuit pas ipso facto, comme l'affirme le paradoxe sceptique - c'est-à-dire la thèse de la normativité intrinsèque de la signification ou de l'intentionnalité -, que les faits sémantiques n'existent pas ${ }^{11}$.

\section{LA SOLUTION SCEPTIQUE (OU L'INTENTIONNALITÉ EST INTRINSÈQUEMENT SOCIALE)}

Dans ce qui suit, je me propose cependant d'admettre le paradoxe sceptique, c'est-à-dire la thèse de la normativité intrinsèque de la signification (ou de l'intentionnalité) dans le but d'examiner la «solution sceptique» préconisée par Kripke (1982) au nom de Wittgenstein (1953) au paradoxe sceptique. L'essence de la solution sceptique consiste à faire valoir que cela n'a pas de sens de suivre une règle «en son for intérieur» ou «en privé». Comme le disait Wittgenstein (1953) dans ses remarques destinées à discréditer l'idée d'un «langage privé»et que cite Kripke (1982), «croire qu'on suit une règle n'est pas la même chose que suivre une règle. S'il était possible de suivre une règle en privé, alors il n'y aurait aucune différence entre le fait de croire qu'on suit une règle et le fait de la suivre». Le paradoxe sceptique affirme que la signification (ou l'intentionnalité) est intrin-

9 Comme l'ont fait valoir indépendamment l'un de l'autre P. Horwich (1998) dans Meaning et F. Dretske (2000) dans Norms, History, and the Constitution of the Mental. La distinction entre la normativité intrinsèque d'une propriété et le fait que l'exemplification d'une propriété peut avoir des conséquences normatives est au cœur de ce que P. Horwich (1998) dans Meaning nomme le « déflationnisme».

10 Je note qu'il existe deux usages du mot «fait»: un usage métaphysique ou substantiel et un usage non métaphysique ou modeste. Dans l'usage métaphysique ou substantiel, ce qui est un fait ne peut pas être une norme (ou une valeur). Mais dans l'usage modeste non métaphysique, rien n'interdit d'affirmer que c'est un fait qu'on doit appliquer le mot «goose» à des oies et à rien d'autre. Dans ce paragraphe, je fais un usage modeste non métaphysique du mot «fait».

11 Pour une discussion détaillée de la stratégie que je recommande pour rejeter le paradoxe sceptique ou la thèse selon laquelle la signification ou l'intentionnalité est intrinsèquement normative, cf. mes articles Les propriétés sémantiques sont-elles intrinsèquement normatives? et Is Meaning Intrinsically Normative? 
sèquement normative. A la question de savoir d'où découle la normativité intrinsèque de la signification (ou de l'intentionnalité), la solution sceptique répond: la normativité de la signification découle des pratiques de la communauté. Les normes sémantiques qui gouvernent la pensée et/ou l'utilisation d'une expression linguistique par un individu résultent des pratiques conceptuelles et/ou linguistiques des membres de la communauté de cet individu. Ce qu'un individu veut dire par son utilisation d'un concept ou d'un mot est constitué par le fait que son usage est conforme à ou concorde avec l'usage des membres de sa communauté. Cet accord est constitutif de l'appartenance de l'individu à sa communauté. Comme le dit Kripke (1982), «un individu qui prétend avoir la maîtrise du concept d'addition sera jugé comme tel par les membres de sa communauté selon que ses réponses à des questions arithmétiques particulières sont en accord avec celles du reste de la communauté dans un nombre suffisant de cas (...) Un individu qui réussit ces tests est admis dans la communauté de ceux qui sont capables d'additionner $\gg$.

Il est tout à fait plausible de supposer que la capacité d'effectuer des additions arithmétiques exactes dépend de la faculté de langage et de la communication verbale. Autrement dit, il est plausible de supposer que les concepts de l'arithmétique sont des concepts «déférentiels». Mais la solution sceptique est supposée s'appliquer à la conceptualité en tant que telle. Un concept se définit notamment par les inférences auxquels il se prête. Posséder un concept, c'est être capable d'effectuer certaines inférences. Or, on peut soutenir que la notion d'inférence (qui suppose l'existence de règles) est une notion elle-même normative. La solution sceptique est donc supposée valoir pour la possession de tout concept quelconque. Elle affirme donc que faute d'appartenir à une communauté d'agents doués d'intentionnalité, un individu serait privé d'intentionnalité ou que l'intentionnalité est une propriété intrinsèquement sociale.

Avant de faire valoir mes raisons de penser que la solution sceptique s'apparente davantage à une «dissolution» du paradoxe sceptique qu'à une solution authentique, je voudrais brièvement mettre en garde contre deux dangers ou deux conséquences potentielles indésirables de la solution sceptique. Premièrement, la question se pose de savoir si la solution sceptique consiste à attribuer l'intentionnalité, des intentions de signifier, des croyances ou des pensées conceptuelles à une communauté. Si tel était le cas, alors je ferais immédiatement observer que cette attribution ne peut, dans le meilleur des cas, qu'être une métaphore. Qu'estce qu'une métaphore? Une métaphore est l'énoncé d'une proposition littéralement fausse grâce à laquelle on communique indirectement certaines propositions vraies. En énonçant, par exemple, «Jacques Chirac est un lion», ce qu'on dit explicitement est faux car un être humain n'est pas un lion. Mais on pourrait, par le biais d'une proposition littéralement fausse, communiquer implicitement ou indirectement certaines propositions vraies sur Jacques Chirac. Affirmer qu'une communauté pense, qu'elle a des intentions ou qu'elle possède des concepts, c'est énoncer une proposition littéralement fausse. En effet, une communauté est privée des capacités cognitives qui accompagnent normalement la possession des concepts et de l'intentionnalité. Il serait mystérieux de conférer l'intentionnalité ou la possession de concepts à une communauté, c'est-à-dire à une entité dépourvue d'un corps permettant de se mouvoir, d'organes permettant de percevoir et d'un cerveau doté d'une mémoire et de capacités de raisonner. On pourrait m'ob- 
jecter qu'une communauté est dotée des cerveaux et des capacités cognitives des individus qui la composent. Mais ce serait commettre une erreur de catégorie: sans doute une communauté est-elle un ensemble d'individus dotés de cerveaux et de capacités cognitives. Mais un ensemble d'individus n'est pas un individu et un réseau connecté de cerveaux n'est pas un cerveau.

Deuxièmement, la solution sceptique soulève la question épineuse de la portée, de l'extension et des limites du concept exprimé par le mot «communauté». La définition de la portée et des limites de la communauté soulève à son tour le spectre du relativisme conceptuel. Dans le cas où la communauté est supposée fixer la règle de l'addition arithmétique, l'extension de la communauté semble s'identifier à l'ensemble des créatures susceptibles de raisonner en conformité avec les lois logiques elles-mêmes. Mais, comme le montre l'histoire des idées scientifiques, différentes communautés à différentes époques ont des croyances différentes.

Considérons succinctement la controverse entre le géocentrisme et l'héliocentrisme en cosmologie ${ }^{12}$. Dans la conception héliocentriste du système solaire, un concept astronomique comme le concept exprimé par le mot français «Terre» fait référence à une planète, c'est-à-dire à un corps qui tourne régulièrement autour du Soleil. Dans la conception géocentriste du monde, le concept exprimé par le même mot fait référence à une entité immobile au centre de l'univers. Si la référence de ce concept est fixée par le système des croyances propres à une communauté historique particulière, alors le risque existe qu'en employant le même mot, les membres de deux communautés historiques différentes ne font pas référence à la même entité. Si la référence du concept géocentriste exprimé par le mot français «Terre » est déterminée par le système de croyances en vigueur dans la communauté des astronomes géocentristes et si la référence du concept héliocentriste exprimé par le même mot français est déterminée par le système des croyances en vigueur dans la communauté des astronomes héliocentristes, alors par le même mot «Terre», un astronome géocentriste et un astronome héliocentriste risquent de ne pas faire référence à la même chose. S'ils ne font pas référence à la même entité, alors on ne peut tout simplement pas comparer leurs croyances. S'ils ne font pas référence à la même entité, ils ne peuvent pas attribuer à une seule et même chose des propriétés différentes et éventuellement incompatibles l'une avec l'autre. Autrement dit, ils ne peuvent pas se contredire. Et nous ne pouvons pas déterminer leurs points d'accord et de désaccord. En effet deux croyances ne peuvent se contredire ou être mutuellement incompatibles l'une avec l'autre que si elles attribuent à une seule et même chose des propriétés mutuellement incompatibles. Or, la conclusion selon laquelle les croyances géocentristes et les croyances héliocentristes sont incomparables et non contradictoires est simplement contredite par la pratique des historiens des sciences qui comparent minutieusement le système des croyances géocentristes au système des croyances héliocentristes.

Quoiqu'il en soit des conséquences potentielles indésirables de la solution sceptique au paradoxe sceptique, je voudrais à présent faire valoir que la solution

12 On peut lire dans le présent paragraphe une critique de la fameuse thèse kuhnienne de l'incommensurabilité entre paradigmes que défendait T.S. Kuhn (1962) dans son classique ouvrage, The Structure of Scientific Revolutions. 
sceptique s'apparente davantage à une dissolution qu'à une solution authentique du paradoxe sceptique. A la thèse méta-arithmétique naïve selon laquelle ce qui justifie ma réponse arithmétique « 125 » à la question arithmétique «Combien font $68+57$ ?», le sceptique opposait l'objection de la finitude. Comme il existe un fossé entre l'ensemble fini des additions que j'ai exécutées et l'ensemble infini des cas auxquels s'applique la règle d'addition, rien ne peut me garantir qu'en répondant «125», je n'ai pas calculé la fonction quaddition et je n'ai pas suivi la règle d'usage du symbole «quus» plutôt que celle du symbole «plus». La première question soulevée par la solution sceptique est de savoir si l'appel à la communauté permet de répondre à l'objection de la finitude. Manifestement, l'ensemble des additions effectuées à un instant déterminé par les membres d'une communauté est un ensemble fini. A moins que l'espèce humaine ne se perpétue indéfiniment, l'ensemble des additions effectuées restera un ensemble fini. Je ne vois donc pas comment l'invocation de l'appartenance à une communauté peut résoudre le problème de la finitude. Ce que me suggère l'idée selon laquelle la reconnaissance des capacités arithmétiques d'un individu par le reste de sa communauté serait constitutive de sa maîtrise du concept exprimé par le symbole «+» et de la règle d'addition, c'est que l'attitude recommandée par le tenant de la solution sceptique est de dissoudre et non de résoudre le problème de la finitude.

A la question de savoir quels sont les faits psychologiques et mentaux susceptibles de servir de conditions de vérité à l'attribution d'une intention de signifier à un calculateur ou à l'attribution de signification à un symbole arithmétique, le calculateur naîf fournissait la solution dispositionnelle. A la réponse dispositionnelle, le sceptique opposait l'objection de la normativité: le problème n'est pas de savoir ce que répondrait le calculateur naïf mais ce qu'il doit répondre. Et à ce problème, la réponse dispositionnelle n'est pas une solution. La question soulevée par la solution sceptique est de savoir si l'appel à la communauté permet de résoudre le problème de la normativité. Manifestement, les membres (les plus éminents) d'une communauté de calculateurs n'ont rien d'autre à offrir que l'ensemble de leurs dispositions à répondre à des questions arithmétiques. S'il existe un fossé entre la disposition d'un individu à répondre à une question arithmétique et ce qu'il doit répondre, ce fossé n'est pas comblé par l'ensemble des dispositions à répondre aux questions arithmétiques de l'ensemble des membres d'une communauté. Entre ce que doivent répondre les membres d'une communauté et ce qu'ils répondraient si la question leur était posée, le fossé reste tout aussi infranchissable.

Selon le sceptique, l'examen de mes dispositions à répondre à une question arithmétique ne permet pas de savoir ce que je dois répondre. Mais les membres de la communauté n'ont eux-mêmes rien d'autre à offrir que leurs dispositions à répondre aux questions arithmétiques. L'examen des dispositions de la totalité des membres d'une communauté permet sans doute de savoir ce qu'ils répondraient à une question arithmétique. Mais il ne permet certainement pas de savoir ce qu'ils doivent répondre. Si ce qui précède est vrai, alors la solution sceptique - la thèse du caractère intrinsèquement social de l'intentionnalité - est plutôt une dissolution du paradoxe sceptique qu'une solution authentique.

Dans ce qui précède, j'ai examiné une thèse qui est sans doute assez populaire chez les historiens et surtout chez les sociologues des sciences - à savoir que, faute d'appartenir à une communauté de personnes conscientes, liées entre elles 
par des contrats, des obligations et des institutions, un individu ne pourrait pas penser. Il serait privé de concepts et d'intentionnalité. Kripke (1982) a fait valoir que cette thèse, que j'ai baptisée «le communautarisme» et qui est notamment d'inspiration wittgensteinienne, peut être invoquée comme la «solution sceptique » d'un paradoxe - le paradoxe sceptique qui lui-même consiste à nier l'existence des faits sémantiques. Wittgenstein (1953) formule cette solution sceptique dans le but de discréditer l'idée même d'un langage privé ou l'idée qu'il est possible de suivre une règle purement privée. Sans aborder vraiment la question de savoir ce que devient l'idée de suivre une règle purement privée, j'ai voulu faire valoir que la solution sceptique n'était pas une solution du paradoxe sceptique. Comme je l'ai indiqué, je suis un réductionniste: j'essaie de réduire l'intentionnalité. Comme Dretske (1981, 1988, 1995) et Fodor (1987), je ne crois pas que les faits sémantiques soient des faits métaphysiques ultimes. Mais je crois qu'il existe des faits sémantiques. Comme l'a dit Fodor (1987), «la sémanticité et l'intentionnalité ne peuvent être des propriétés réelles des choses qu'en vertu de leur identité avec (ou en vertu de leur dépendance systématique par rapport à) des propriétés qui ne sont elles-mêmes ni intentionnelles ni sémantiques ». Donc, de mon point de vue, le paradoxe sceptique est un paradoxe puisqu'il nie l'existence des faits sémantiques. Comme je ne crois pas que la solution sceptique soit une solution authentique, ma recommandation consiste à répudier la thèse selon laquelle l'intentionnalité (ou la signification) est intrinsèquement normative. Je ne crois pas à la normativité intrinsèque de l'intentionnalité. Autrement dit, je répudie l'affirmation selon laquelle l'essence de l'intentionnalité réside dans le pouvoir de représenter ce qui doit être - le devoir être - par opposition à ce qui est. La sémanticité n'est pas intrinsèquement normative. La sémanticité consiste dans le pouvoir de représenter des choses et des états de choses réalisés, possibles et impossibles. Le jour où nous aurons vraiment compris en quoi consiste le pouvoir de représenter des états de choses dont certains sont des faits, nous aurons les moyens d'élucider le pouvoir de représenter des normes.

Institut Jean Nicod

CNRS-EHESS-ENS, Paris

\section{RÉFÉRENCES}

A.J. Ayer, Language, Truth and Logic (1st edition, 1936, $2^{\text {nd }}$ edition, 1946), New York, Dover, 1952.

T. Burge, Individualism and the Mental, in P. French, T. Uehling, Jr. \& H.K Wettstein, (eds.) Midwest Studies in Philosoophy, vol. IV, Minneapolis, University of Minnesota Press, 1979.

D. Davidson, Essays on Actions and Events, Oxford, The Clarendon Press, 1980.

D. Davidson, Inquiries into Truth and Interpretation, Oxford, The Clarendon Press, 1984.

D. Dennett, The Intentional Stance, Cambridge, Mass., MIT Press, 1987.

F. Dretske, Knowledge and the Flow of Information, Cambridge, Mass., MIT Press, 1981.

F. Dretske, Explaining Behaviour, Cambridge, Mass., MIT Press, 1988.

F. Dretske, Naturalizing the Mind, Cambridge, Mass., MIT Press, 1995.

F. Dretske, Norms, History, and the Constitution of the Mental, in F. Dretske, Perception, Knowledge and Belief, Cambridge, Cambridge University Press, 2000. 
M. Dummett, Truth, in M. Dummett, Truth and Other Enigmas, Cambridge, Harvard University Press, 1978.

J.A. Fodor, Psychosemantics, the Problem of Meaning in the Philosophy of Mind, Cambridge, Mass., MIT Press, 1987.

J. Haugeland, Mind Design, Montgomery, VT, Bradford, MIT Press, 1981.

P. Horwich, Meaning, Oxford, Oxford University Press, 1998.

P. Jacob, What Minds Can Do, Cambridge, Cambridge University Press, 1997.

P. Jacob, Pourquoi les choses ont-elles un sens?, Paris, Odile Jacob, 1997.

P. Jacob, Les propriétés sémantiques sont-elles intrinsèquement normatives?, in R. Boudon, P. Demeulenaere \& R. Viale (dir.) L'Explication des normes sociales, Paris, PUF, 2001, pp. 259-278.

P. Jacob, Is Meaning Intrinsically Normative?, in Moulines, C.U. \& Niebergall, K-G. (eds.) Argument and Analysis (à paraître).

S. Kripke, Wittgenstein on Rules and Private Language, Oxford, Blackwell, 1982.

T.S. Kuhn, The Structure of Scientific Revolutions, Chicago, The University of Chicago Press, 1962, 1970.

J. McDowell, Mind and World, Cambridge, Harvard University Press, 1994.

H. Putnam, The Meaning of 'Meaning', in H. Putnam, Mind, Language and Reality, Philosophical Papers, vol. II, Cambridge, Cambridge University Press, 1975.

J. Searle, Intentionality. An Essay in the Philosophy of Mind, Cambridge, Cambridge University Press, 1983.

J. Searle, Rationality in Action, Cambridge, Mass., MIT Press, 2001.

L. Wittgenstein, Philosophical Investigations, Oxford, Oxford University Press, 1953. 